\title{
Artículos
}

\section{El peso de los factores contextuales en la asistencia a la secundaria entre los adolescentes costarricenses según la información censal}

\section{The relevance of contextual factors on secondary school attendance among Costa Rican adolescents according to census information}

\author{
Gilbert Brenes Camacho*
}

\section{Resumen}

El abandono del sistema escolar es un problema social común en América Latina. Se propone un análisis de la asociación entre la asistencia a la educación secundaria entre los jóvenes en edad escolar en Costa Rica y las variables agregadas que describen las características de las áreas en que residen estas personas, con el fin de aproximarse a los posibles "efectos de barrio". Se utiliza un modelo logístico multinivel con datos censales. Se encuentra una asociación positiva entre la asistencia a la educación secundaria y el nivel socioeconómico tanto del joven como del barrio. Se observa también que los jóvenes con discapacidad, los nicaragüenses, los que migraron recientemente dentro del pais, y sobre todo aquellos que están laborando, tienen menor probabilidad de asistir a un centro de enseñanza. Los hallazgos refuerzan la idea de que las políticas públicas para la educación tienen que tomar en cuenta el contexto social en el que vive el joven para mantenerlo estudiando.

Palabras claves: asistencia a la educación; efecto de barrio; nivel socioeconómico; Costa Rica.

\begin{abstract}
High school abandonment is a common problem in Latin America. The article analyzes the association between high school attendance among school-age youngsters and aggregated variables that describe the characteristics of areas where these

* Profesor e investigador, Universidad de Costa Rica, Centro Centroamericano de Población y Escuela de Estadística. Dirección postal: Ciudad Universitaria Rodrigo Facio Brenes, San José, San Pedro, Costa Rica. Correo electrónico: gbrenes@ccp.ucr.ac.cr; gilbert.brenes@ ucr.ac.cr ORCID: https://orcid.org/0000-0002-3163-7018
\end{abstract}


persons live, in order to assess "neighborhood effects". The analysis estimates a multi-level logistic model using census data. There is a positive association between the probability of attending secondary education and the socio-economic status of both the young person and the neighborhood. Young people with disabilities, those born in Nicaragua or who moved to another county within the country, and above all those who are working are less likely to attend an educational institution. Findings stress the idea that educational policies should take into account the social context in which the student lives in order to keep him studying.

Keywords: high school attendance; neighborhood effect; socioeconomic status; Costa Rica.

\section{Introducción}

La no asistencia a la educación formal entre los jóvenes es un problema que viene aquejando a los sistemas educativos en América Latina (Espíndola y León, 2002; Terigi, 2009). En Costa Rica, entre 1950 y 1980, se observó una tendencia de creciente cobertura de la población joven por parte del sistema educativo. Sin embargo, la crisis económica de inicios de la década de los ochenta detuvo esa tendencia y dificultó la retención de los jóvenes en la secundaria (PEN, 2005, 2008, 2011, 2013). La literatura psicológica y sociológica reconoce que la permanencia en el sistema se explica por determinantes individuales y familiares (Arguedas y Jiménez, 2008; Berns, 2012; Espíndola y León, 2002; Fernández, 2009; Montero, Rojas, Zamora y Rodino, 2012; Román, 2009); adicionalmente, hay evidencia creciente de que los factores de la comunidad también pueden determinar el acceso de los jóvenes a la educación (Hallinger y Murphy, 1986; Patterson, 2008; Rendón, 2014; Wodtke, Harding y Elwert, 2011).

El objetivo principal del artículo es mostrar si hay características contextuales de las comunidades costarricenses -el denominado "efecto de barrio" o "neighborhood effect"- que estén asociadas estadísticamente con la asistencia al sistema educativo, controlando por las características individuales de los jóvenes y de sus hogares, y usando datos censales. Los resultados pueden ser útiles para planear estrategias de detección de jóvenes excluidos del sistema escolar mediante la utilización de perspectivas comunitarias más eficientes y políticas públicas a nivel comunitario. 


\section{Relación entre asistencia a la educación y características de la comunidad}

La relación entre los niños y los jóvenes, por un lado, y las instituciones educativas como agentes socializadores por otro, está mediada por factores de muy diversos niveles: desde la familia, la escuela y la comunidad en los niveles más cercanos a los estudiantes, hasta las políticas públicas, el contexto sociocultural o el sistema económico imperante en una sociedad (Berns, 2012; Bronfenbrenner, 2005). Por consiguiente, la asistencia al sistema escolar está determinada por múltiples factores. Las teorías que ligan al contexto del estudiante con su desempeño educativo se fundamentan generalmente a partir del concepto de capital social (Bourdieu, 1986; Coleman, 1988; Putnam, 1993). El capital social se refiere a todos los recursos disponibles para una persona o conjunto de personas que surgen de sus relaciones sociales con otras personas y organizaciones. Para Bourdieu (1986) en particular, estas relaciones sociales son desarrolladas y mantenidas para poder generar beneficios actuales o futuros, y por eso se consideran como "capital"; además, todo tipo de capital (económico, social, cultural) es intercambiable ("fungible") con el fin de producir los beneficios esperados.

Tal y como lo señala Portes (2000), el capital social en su operacionalización se ha definido de distintas formas de acuerdo con los objetivos de la investigación; señala tres vertientes: el capital social como fuente de beneficios mediados por la familia, como fuente de control social, y como fuente de beneficios que surgen de relaciones no familiares. La primera definición fomentó la investigación sobre el papel de la familia en el éxito escolar (Dika y Singh, 2002). Según esta literatura, el estudiante tiene más posibilidades de mantenerse en el sistema escolar y obtener mejores calificaciones si cuenta con el apoyo familiar, tanto en términos afectivos como en términos de disponibilidad de recursos económicos (por ejemplo, Moreira-Mora, 2007; Trejos, 2010; Tyler y Lofstrom, 2009). Además, para Coleman (1988), la familia es la primera instancia del control social que constituye el capital social, pues establece las normas explícitas o implícitas que se cumplen a través del sentido del honor o pertenencia y la necesidad de mantener la cohesión del grupo familiar. Por el contrario, para Bourdieu (Bourdieu, 1986; Dika y Singh, 2002; Portes, 2000), el capital social de un individuo está constreñido por su posición de clase, pues se entiende que el capital social comprende una serie de recursos para mantener la reproducción del sistema. Desde este punto de vista, los estudiantes de clases dominantes tendrían mayor acceso a recursos surgidos de sus interacciones sociales que los estudiantes provenientes de otras clases sociales. 
Desde estas perspectivas, la familia es un ámbito primordial de normas y accesos diferenciales a los recursos, pero no es el único; la comunidad surge entonces como el siguiente ámbito para delimitar e intercambiar los recursos y normas del capital social. En los ejemplos que cita Coleman (1988) de la comunidad como proveedora de ese capital social, los lazos no familiares se entienden como parte de una "gran familia". La comunidad establece límites respetados por sus miembros para asegurar su propio bienestar: "Una comunidad con normas fuertes y efectivas sobre el comportamiento de los jóvenes los puede mantener alejados de la buena vida" (Coleman, 1988, p. S105). La delimitación de qué es una comunidad para que ésta sea fuente de capital social varía de acuerdo con los recursos requeridos por los individuos. Por ejemplo, para Portes y MacLeod (1996), el capital social de los niños inmigrantes está relacionado con su identificación y nexos con su comunidad étnica; para Coleman (1988), una comunidad puede surgir de organizaciones sociales apropiables: organizaciones formales o informales en las que sus miembros encuentran vías para conseguir objetivos en común.

Aunque no toda comunidad se puede definir por su delimitación geográfica (Agnew, 1989), la distancia física está altamente asociada a la distancia social; cuanto más cerca esté un individuo de otros miembros de un colectivo, se esperaría que tenga más cantidad de interacciones con éstos. El "barrio" surge entonces como un ámbito para el desarrollo del capital social, a tal punto que se genera el concepto de capital comunitario, el cual comprende el desarrollo de los distintos capitales (económico, social, cultural, ambiental) dentro de una comunidad definida generalmente por límites geográficos, que buscan la sostenibilidad y resiliencia de la misma (Callaghan y Colton, 2008). El factor político es importante dentro de la configuración del capital comunitario, si los gobiernos y las organizaciones locales están involucrados en el planteamiento y ejecución de políticas educativas (Terigi, 2009). Este concepto de capital comunitario se asocia con el modelo bioecológico de Bronfenbrenner (2005), al comprender que las relaciones sociales en el micronivel se facilitan y desarrollan en varios niveles de agregación, desde la comunidad física hasta la sociedad en general.

En el contexto mexicano, Solís y Puga (2011) delinean los mecanismos que pueden ligar el nivel socioeconómico del lugar de residencia con el logro educativo. Algunos de estos mecanismos se relacionan con características de las instituciones educativas -la calidad y disponibilidad de las mismas en los lugares de la localidad donde se vive-, mientras que otras se asocian con las características físicas y sociales del lugar -como las normas sociales y expectativas posiblemente provenientes de las familias que habitan la localidad, o las restricciones físicas a las actividades rutinarias que le permiten 
al estudiante proseguir sus estudios efectivamente-. Si se entrelazan estos tipos de factores, se podría considerar que las características del contexto físico del joven pueden facilitar su interacción con su centro educativo, en particular si éste es visto como parte de la comunidad (Bronfenbrenner, 2005). Hallinger y Murphy (1986) encuentran en Estados Unidos que las instituciones educativas ubicadas en áreas de nivel socioeconómico bajo tenían menos "efectividad" administrativa que otras instituciones. Domingos (1989) y Lizasoain, Joaristi, Lukas y Santiago (2007) describen interacciones entre el nivel económico de las instituciones o las comunidades y el de las familias para predecir el rendimiento académico. Estos autores fundamentan su análisis en el marco teórico de Coleman (1988); argumentan que los centros educativos de nivel socioeconómico alto se benefician al poder contratar personal más calificado, pero también de un mayor interés de los padres de familia en interactuar con la institución. Otras investigaciones analizan el "efecto de barrio" sobre el rendimiento escolar entre estudiantes de minorías; las asociaciones varían según la comunidad étnica que se analice (Eamon, 2005; Pong y Hao, 2007; Portes y McLeod, 1996). En ellas, se emplea el argumento de que las ventajas de las interacciones interpersonales (particularmente el involucramiento familiar) favorece a los estudiantes en su desempeño escolar, pero también se plantea que los problemas de rendimiento académico entre hijos de inmigrantes pueden ser explicados por barreras erigidas por el capital social de sus compañeros "nativos". Coleman (1988) acuña el término "closure" ("cerrazón") para explicar que las redes sociales altamente cohesionadas pueden beneficiar a los miembros que están dentro de la red, pero se convierten en círculos cerrados con barreras para que las personas ajenas a la red puedan aprovechar los recursos del conglomerado. En este caso, los "barrios étnicos" podrían favorecer a los estudiantes que pertenecen a la comunidad, pero desfavorecer a los que no se identifican con ella.

En el caso específico de la permanencia en el sistema educativo, a partir de un diseño cuasi experimental, Kling, Liebman y Katz (2007) concluyen que la migración hacia barrios con un mejor bienestar socioeconómico favorece la retención de las mujeres -pero no de los hombres- en las instituciones educativas. Wodtke, Harding y Elwert (2011) operacionalizan la desventaja económica de los barrios utilizando datos censales sobre pobreza, beneficios sociales, tipos de hogares, clima educativo y estructura ocupacional. Estos autores encuentran un "efecto de dosis": cuanto mayor sea el tiempo durante el que se residió en las áreas con mayor desventaja, mayor es la probabilidad de no graduarse de la secundaria. Patterson (2008) también encuentra asociación entre el nivel de pobreza y la probabilidad de graduarse en Estados Unidos según datos censales; la asociación es más fuerte entre 
la población caucásica que entre la población afroamericana o la hispana. Rendón (2014) muestra que el porcentaje de graduados de secundaria en un barrio está asociado con una menor probabilidad individual de terminar la secundaria, pero la relación está mediada por las características de la institución educativa. La explicación teórica de sus hallazgos por parte de estos últimos autores se centra más en las desventajas del contexto físico y de pobreza de los jóvenes, y su incidencia en su rendimiento como estudiantes.

\section{Asistencia al sistema educativo en América Latina y Costa Rica}

Las investigaciones latinoamericanas sobre la permanencia de los estudiantes en el sistema educativo reconocen que el fenómeno se debe a una multiplicidad de factores económicos, sociales y personales (Espíndola y León, 2002; Fernández, 2009). En Uruguay, Cardozo (2010) cita las razones que aportan los estudiantes para desafiliarse de la educación media. Estas razones incluyen la necesidad de trabajar, el desinterés por el estudio, el rendimiento académico bajo o el inicio de la procreación; aclara que la importancia relativa de estas razones varía de acuerdo con la edad en la que se da el abandono al sistema escolar. Román (2009) aduce que se tienen que comprender los factores exógenos (características individuales y familiares de los estudiantes) y endógenos (pedagogía, características físicas y educativas del centro educativo, disponibilidad de docentes) para comprender el fracaso escolar; ella encuentra que en Chile, los jóvenes de 14 a 15 años, sobre todo hombres de bajos ingresos, tienen mayor riesgo de no asistir a la educación formal. Alcázar (2009) describe patrones similares en Perú. Encuentra además que el empezar una unión conyugal o procrear hijos, así como tener malas relaciones con los progenitores, aumentan el riesgo de dejar las aulas. Cuando se describen las razones de no estudiar, se citan tanto las relacionadas con necesidades económicas como el desinterés por la educación o los problemas de bajas calificaciones. La inserción en el mercado laboral también está asociada a la no asistencia a la educación, aunque la autora señala que no se puede definir claramente cuál de los dos fenómenos incide directamente en el otro, pues la secuencia temporal de los dos eventos (trabajo y abandono del sistema escolar) pueden darse en forma diferente. Fernández (2010a, 2010b) argumenta para Uruguay que los jóvenes experimentan distintas transiciones en las que a veces la inserción laboral precede a la desafiliación educativa, y a veces la sucede, pero que la acumulación de la desafiliación y el acceso precario al mercado laboral, sobre todo sin acceso a la seguridad social, conforman un mecanismo claro de exclusión social 
para los jóvenes. Aristimuño (2009) y Fernández (2009) explican que en Uruguay los estudiantes que tienen una mala opinión sobre estudiar tienen menos probabilidades de estar asistiendo a la secundaria; asociado a este fenómeno, con datos longitudinales, Fernández (2009) muestra que los jóvenes que obtenían malas calificaciones en la primera entrevista, tenían mayores probabilidades de dejar de estudiar en las entrevistas subsecuentes.

Las características del contexto también están determinadas por la segregación o la concentración de poblaciones caracterizadas como "minoritarias" por la sociedad dominante. La relación entre el logro educativo y el capital social de las comunidades migrantes descritas en Estados Unidos (Eamon, 2005; Pong y Hao, 2007; Portes y McLeod, 1996) tiene sus variantes en el caso latinoamericano. Si bien es cierto que las razones para no asistir a la secundaria que dan los jóvenes inmigrantes son parecidas a las que dan los jóvenes nacionales (Rodríguez Romero, 2014; Tijoux, 2013), los jóvenes inmigrantes se enfrentan a situaciones particulares que incrementan su probabilidad de abandono del sistema. Los jóvenes nicaragüenses en secundarias costarricenses están más expuestos a la necesidad de desplazarse en forma más constante (Rodríguez Romero, 2014). Los niños y jóvenes inmigrantes peruanos cumplen una función particular en las áreas urbanas pauperizadas de Santiago de Chile. Su incorporación al sistema educativo permite mantener abiertas a las instituciones escolares que experimentan decrecimientos en su población estudiantil; sin embargo, los estudiantes se enfrentan a tratos discriminatorios no sólo en las instituciones, sino en los mismos barrios segregados, lo cual les puede afectar en su trayectoria educativa (Tijoux, 2013).

Un contexto de discriminación y poco apoyo es el que enfrentan los estudiantes indígenas en los sistemas escolares latinoamericanos. Aparte de sus propias desventajas socioeconómicas - mayores necesidades de trabajar o de participar en labores domésticas, residir en zonas alejadas poco pobladas- (Murillo López, 2005), al igual que los inmigrantes, la comunidad indígena es percibida como ajena a los objetivos del proceso educativo. Esta situación se agrava cuando el estudiante tiene a la lengua indígena como su lengua primaria de desenvolvimiento dentro de su comunidad, pero el sistema educativo le imparte lecciones en el idioma oficial. En Perú (Cueto, 2004), los estudiantes cuya lengua materna no es el castellano obtienen menores calificaciones en pruebas estandarizadas y tienen mayor probabilidad de dejar las aulas. No obstante, la literatura documenta ejemplos exitosos en México y Perú, en donde las comunidades o las autoridades promueven la educación bilingüe (Alonso Meneses y Ángeles Salinas, 2014; Baronnet, 2013), característica que promueve la asistencia del joven (Rodríguez Lozano, 2012). 
En Costa Rica, la mayoría de las investigaciones sobre la no asistencia a la secundaria se ha centrado en aspectos del sistema escolar y en factores individuales de los estudiantes. Entre los factores individuales más citados se encuentran la desmotivación o falta de interés hacia el sistema formal, los problemas de aprendizaje, el bajo rendimiento en las evaluaciones, la cantidad de cursos y responsabilidades académicas, y el miedo a la transición entre la primaria y la secundaria, entre otros (Arguedas-Negrini y JiménezSegura, 2008; Castro-Valverde, 2006; Herrera-Vargas, 2012; Moreira-Mora, 2007; Ruiz-Guevara, Castro-Pérez y León-Sáenz, 2010).

Son pocos los estudios en Costa Rica que investigan la asociación entre el abandono del sistema escolar y los factores contextuales. La mayoría se centra en el papel que juega la familia en el rendimiento escolar. Una serie de investigaciones muestra que la asistencia a la escuela y el colegio es menor en hogares con un nivel socioeconómico precario porque los jóvenes tienen que insertarse en el mercado laboral, descuidando las responsabilidades del estudio (Castro-Valverde, 2006; Herrera-Vargas, 2012; Moreira-Mora, 2007). Dentro de los estudios que analizan el nivel socioeconómico como determinante, cabe resaltar aquellos que miden la efectividad de programas públicos destinados al combate a la pobreza. El programa Avancemos -basado en transferencias monetarias a familias de bajos ingresos- ha cumplido con su objetivo de aumentar la retención del estudiantado en la educación secundaria (Vargas y Slon, 2012). La educación de los progenitores predice también el logro académico de los jóvenes. El denominado "clima educativo del hogar" explica el rendimiento estudiantil más allá de las limitaciones económicas (Trejos, 2010); padres con menos años de escolaridad tienen menos posibilidades de apoyar a sus hijos en las responsabilidades del hogar (MoreiraMora, 2007). Con una perspectiva más demográfica, Li, Dow y Rosero-Bixby (2014) encuentran que, para cohortes nacidas en los setenta, tener más hermanos disminuía la probabilidad de mantenerse en el sistema escolar; para las cohortes más recientes, esta asociación prácticamente desaparece. Otros estudios resaltan las diferencias geográficas en las tasas de deserción estudiantil (PEN, 2011, 2013; Trejos, 2010).

No obstante, salvo Brenes-Camacho (2014), ningún autor ha tratado de relacionar en forma cuantitativa el abandono escolar con las características propias de la comunidad. Este investigador encuentra que cuanto mayor es el porcentaje de personas con discapacidad (con limitaciones funcionales), mayor es la tasa de deserción medida a nivel de institución escolar. Además, reporta que cuanto mayor es el porcentaje de hogares con tanque de agua caliente (calentador) en el barrio en el que se ubica una institución educativa, menor es la tasa de deserción en la institución. En Costa Rica, la posesión 
de tanque de agua caliente es un indicador de nivel socioeconómico alto, pues este bien es relativamente caro para el nivel adquisitivo promedio del costarricense. Ambas asociaciones tienen las direcciones esperadas. Una limitación del análisis de Brenes-Camacho (2014) es que la unidad de análisis es la institución educativa, y las covariables están medidas como indicadores agregados que se refieren a la comunidad en la que está ubicada la secundaria. Por consiguiente, no se puede diferenciar si la asociación ocurre en el nivel agregado y refleja posibles "efectos de barrio", o si se debe enteramente a dinámicas individuales que se mantienen cuando los datos se agregan.

Cabe aclarar que, en la mayoría de las investigaciones citadas tanto para América Latina como para el caso costarricense, los datos recopilados son transversales, por lo que el indicador medido es la no asistencia al sistema escolar, a pesar de que los autores utilizan conceptos tales como deserción o abandono. Sólo Fernández (2009) cuenta con datos longitudinales, pero prefiere usar el término desafiliación en lugar de deserción para explicar que la no asistencia al sistema educativo surge no sólo de un fenómeno individual de incumplimiento de normas, sino también de procesos excluyentes por parte del Estado para incentivar la permanencia del joven.

\section{Datos y métodos}

Los datos provienen del X Censo Nacional de Población y VI de Vivienda de Costa Rica, cuyo trabajo de campo se efectuó entre el 30 de mayo y el 3 de junio de 2011 (INEC, 2011). Para tener más eficiencia en el procedimiento estadístico, se seleccionaron al azar 1310 UGM (Unidades Geográficas Mínimas) de un total de 13100 UGM que tenían al menos 10 personas entre los 12 y los 17 años de edad que aprobaron la primaria pero que aún no habían aprobado la secundaria académica o técnica. Las UGM son conjuntos de viviendas geográficamente aglomeradas, generalmente delimitadas por calles u otros hitos geográficos; fueron construidas por el Instituto Nacional de Estadística y Censos (INEC) de Costa Rica para la planeación del trabajo de campo de los censos de población. La UGM puede ser considerada como una operacionalización estadística del "barrio" en el que vive el joven. Se decidió eliminar UGM con menos de 10 jóvenes con las características deseadas para evitar problemas de estimabilidad en modelos de regresión con variable dependiente cualitativa. La unidad estadística básica es el joven de 12 a 17 años con primaria concluida, pero sin graduarse de secundaria. De esta forma se asegura que el análisis infiere a la población que normativamente debería estar 
asistiendo a las instituciones de educación secundaria (colegios). En la muestra de UGM se tomaron a todos los jóvenes que cumplieran con los criterios de inclusión antes esbozados, por lo que se cuenta con una muestra total de 27987 personas.

La variable dependiente es la asistencia al sistema educativo. Es una variable dicotómica igual a 1 si la persona de dicha edad estaba asistiendo a la educación regular según la pregunta censal, condicional a que tiene ya aprobado el último año de la educación primaria (seis años). Esta operacionalización tiene varias limitaciones. En primer lugar, no se puede asegurar que esta persona no regresará al siguiente año a la secundaria. En segundo lugar, no se puede saber si la persona se matriculó en la secundaria y desertó, o si nunca ingresó al séptimo año de educación formal. Hay entonces varias trayectorias posibles que pueden conllevar a que un joven no esté asistiendo a la educación secundaria (Cardozo, 2010; Fernández, 2010b) en un momento dado: a) nunca se matriculó en la secundaria; b) se matriculó al inicio del año lectivo en la secundaria y desertó permanentemente del sistema educativo, por lo que no regresaría a estudiar; $c$ ) en el momento censal no estaba asistiendo temporalmente a clases de la secundaria por motivos personales (por ejemplo, trabajo temporal, situación económica de la familia, enfermedad, etc.), pero piensa volver a matricularse en el siguiente año lectivo; o $d$ ) abandonó el sistema de educación formal que está definido por fechas de inicio y final del calendario escolar, y planea matricularse en la educación abierta (educación en institutos de tutoría en los que el estudiante asume el estudio con su propio ritmo), donde hay más flexibilidad en las fechas de inicio y finalización de los estudios. Las investigaciones basadas en estadísticas oficiales (PEN, 2005, 2008, 2011, 2013) sugieren que la segunda trayectoria (de deserción) es la más probable, pues hay una alta deserción a mitad del primer año de la educación secundaria; sin embargo, no se puede asegurar que sea la única trayectoria.

Las variables independientes se dividieron en variables individuales y variables de barrio. Las variables individuales se refieren a características sociodemográficas de los jóvenes y sus hogares (sexo, años de escolaridad del jefe del hogar, nivel socioeconómico, número promedio de menores de edad en el hogar, si el hogar tiene al menos un emigrante, condición laboral, condición de discapacidad, si la persona nació en Nicaragua, ${ }^{1}$ si es indígena, y si la persona inmigró al lugar de residencia durante los últimos cinco años). Dado que el censo de Costa Rica no recolecta información sobre el ingreso del hogar, el nivel socioeconómico se operacionalizó construyendo un índice aditivo

1 Nicaragua es el principal país de origen de la comunidad inmigrante en Costa Rica.

Estudios Demográficos y Urbanos, vol. 34, núm. 2 (101), 2019, pp. 395-425

doi: http://dx.doi.org/10.24201/edu.v34i2.1636 
simple con un puntaje mínimo de 0 y un puntaje máximo de 8 ; el índice suma un punto por cada una de las siguientes condiciones: si la vivienda no es considerada tugurio (construida con material de desecho), si la tenencia de la vivienda no es en precario, si no hay hacinamiento en la vivienda, y tenencia de vehículos, televisión por cable, tanque de agua caliente, computadora e internet. Utilizando la Encuesta Nacional de Hogares de 2011, que sí contiene información sobre el ingreso del hogar, se encontró que el coeficiente de correlación lineal entre el índice y el ingreso total del hogar es de 0.5046. Además, el alfa de Cronbach del índice aditivo es de 0.665.

En cuanto a las otras variables independientes, la discapacidad se operacionaliza como una variable dicotómica que sería igual a 1 si la persona menciona que tiene al menos una de las limitaciones funcionales enumeradas en el censo (limitación intelectual -retardo mental, síndrome de Down-, mental-enfermedad bipolar, esquizofrenia, etc.--, visual, auditiva, limitación para hablar, para caminar o para utilizar brazos y piernas). Otra característica importante incorporada como variable independiente es si el joven se declara económicamente activo (ocupado o desempleado buscando empleo). Una clara limitación de esta variable es su carácter endógeno en el modelo debido a la causalidad inversa: no se puede delimitar si la persona joven dejó los estudios porque estaba trabajando, o si empezó a trabajar debido a que había dejado los estudios. Sin embargo, dado que el objetivo principal de la investigación es determinar la asociación del abandono escolar con las variables contextuales, la incorporación de la variable individual de si la persona está económicamente activa o no, cumple la función de reducir posibles sesgos de la variable omitida.

Las variables de barrio son agregaciones de la mayoría de las variables individuales: el índice económico promedio del barrio, la escolaridad promedio de los jefes del hogar, la proporción de hogares con emigrantes, la proporción de jóvenes trabajando, y los porcentajes de personas (no sólo jóvenes, sino también adultos) que son nicaragüenses, indígenas o migrantes internos durante los últimos cinco años. El índice económico promedio y la escolaridad promedio son operacionalizaciones similares a las usadas por Solís y Puga (2011). Se decidió separarlos para diferenciar entre un indicador exclusivo de afluencia física (el índice económico), y uno que se refiriera únicamente al clima educativo del barrio para diferenciar la creación de capital social a partir de recursos financieros (consumo) de la creación del capital social basado en el conocimiento. Para los últimos tres porcentajes, se juzgó relevante considerar a toda la población y no sólo a la población joven, para medir mejor las características sociales del lugar de residencia. Por ejemplo, un hijo de inmigrantes nicaragüenses es considerado nativo de 
Costa Rica, pero puede vivir en un barrio con una población predominantemente migrante.

Adicionalmente, se clasifica a la UGM como rural o urbana de acuerdo con las definiciones censales. Es importante aclarar que las variables que se refieren a la concentración de poblaciones particulares -inmigrantes, indígenas, migrantes internos- responden a la literatura que describe que la construcción de capital social entre poblaciones denominadas como "minoritarias" es diferente a las del grupo dominante, pues es comparativamente más limitado el acceso que tienen estos grupos a recursos suficientes que puede aprovechar el estudiante para mantenerse en el sistema educativo (Bourdieu, 1986; Coleman, 1988; Portes y MacLeod, 1996). En el contexto latinoamericano específicamente, el joven "minoritario" (inmigrante, indígena, migrante rural) se enfrenta a dificultades para seguir estudiando no sólo por sus características individuales, sino también por habitar en contextos sociales en los que los procesos de exclusión (por ejemplo, discriminación, segregación residencial, infraestructura física limitada en zonas alejadas) se exacerban.

Dado que el objetivo principal es medir dinámicas en el nivel de comunidad, se propone utilizar modelos logísticos multinivel. Siguiendo a Wong y Mason (1985), el modelo logístico multinivel o jerárquico se podría describir de la siguiente forma. Sea $i$ el subíndice para la microunidad (el joven de 12 a 17 años), $j$ el subíndice para la macrounidad (las comunidades) y $k$ el subíndice para cada una de las variables predictoras. Además, sea $Y_{i j}$ la variable dependiente (que es igual a 1 si la persona $i$ en el área $j$ no está asistiendo a clases), y $p_{i j}=P\left(Y_{i j}=1\right)$, entonces, se supone que $Y_{i j}$ es una variable aleatoria Bernoulli con probabilidad igual a $p_{i j}$. En el primer nivel de análisis (el del individuo):

$$
\log \left(\frac{p_{i j}}{1-p_{i j}}\right)=\beta_{j 0}+\beta_{j 1} X_{j 1}+\ldots+\beta_{j K} X_{j K}
$$

El coeficiente $\beta_{j k}$ se refiere al coeficiente de regresión de la variable $X_{k}$ para la microunidad $i$ y la macrounidad $j$.

En el siguiente nivel, la variabilidad de los $J$ coeficientes para las microunidades para la variable $k$ se explica con otra serie de variables independientes de nivel macro $G_{j k}$ de la siguiente forma:

$$
\beta_{j k}=\eta_{0}+\eta_{1} G_{j 1}+\ldots+\eta_{K} G_{j K}+a_{j k}
$$


El coeficiente $\eta_{k}$ corresponde al coeficiente de regresión para la variable $G_{k}$, y el término $a_{j k}$ es el término aleatorio para la macrounidad $j$ y covariable $k$. Se supone que $a_{j k}$ se distribuye normalmente con media 0 y varianza $\gamma_{k k}$.

En la práctica, sólo se tiene una serie de coeficientes $\beta_{k}$ tanto para las variables del primer nivel como las del segundo nivel. El modelo multinivel consiste en estimar la varianza $\gamma_{k k}$ para analizar cuánto variarían todos los posibles coeficientes $\beta_{j k}$ que se puedan estimar dentro de cada macrounidad $j$. Si no hay variables $G_{j k}$, aún se estima la variabilidad del término $\eta_{0}$. A este modelo se le conoce como modelo de interceptos aleatorios. Si se incorporan las variables $G_{j k}$, se tiene un modelo con pendientes aleatorias.

En el análisis específico de los datos de Costa Rica se estiman cuatro modelos. El primero es un modelo nulo que tiene únicamente el intercepto general y la varianza del nivel de comunidad. El segundo modelo incorpora sólo las variables individuales, y el tercero las variables comunitarias únicamente. Por último, el cuarto modelo se estima con todas las variables individuales y contextuales. Esta estrategia secuencial permite analizar si las asociaciones que se encontraron en el estudio previo con comunidades como unidades estadísticas (Brenes-Camacho, 2014) se debían a sesgos de la variable omitida. Todos estos modelos se consideran modelos de interceptos aleatorios. Se estimó adicionalmente un modelo de pendientes aleatorias para explorar posibles variaciones en el coeficiente de la variable de tenencia de televisión por cable.

\section{Resultados}

De acuerdo con el censo de 2011, el 84\% de los jóvenes entre 12 y 17 años con estudios primarios aprobados (y viviendo en UGM con al menos 10 jóvenes con las mismas características) estaba asistiendo a la educación secundaria. El porcentaje es relativamente alto para las estadísticas oficiales (PEN, 2013) y su magnitud se explica porque no se considera a las personas extraedad (más de 17 años) que no han concluido la secundaria y no están tomando lecciones regulares.

Las demás características individuales exploradas en el análisis reflejan características demográficas típicas de Costa Rica: la escolaridad promedio está entre siete y ocho años aprobados, cerca de dos menores por hogar (relacionado con la baja fecundidad en el país) y una baja proporción de población indígena. Los porcentajes de jóvenes con ciertas características demográficas (hogares con emigrantes internacionales, inmigrantes nicaragüenses, migrantes internos) es menor que los porcentajes promedio de sus respectivos 
barrios. Esta diferencia se debe a que estas características demográficas son más comunes entre los adultos jóvenes que entre los adolescentes.

Se resalta además que el valor medio del índice económico de los hogares de los jóvenes (4.1) es similar al promedio de los índices de barrio (4.2). El índice varía entre 0 y 8 puntos, y se construyó de tal forma que discriminara los extremos de la distribución. Es importante entonces resaltar que los valores de este índice no tienen una interpretación clara, pero se construyó para medir la asociación entre el nivel socioeconómico individual y contextual con la asistencia a la secundaria (véase el Cuadro 1).

Seguidamente se estimaron los cuatro modelos descritos en la sección metodológica. La primera información valiosa para definir si existe algún "efecto" del contexto es la estimación de la varianza de nivel agregado. La Gráfica 1 ilustra las varianzas estimadas que corresponden al nivel del barrio y sus intervalos de confianza. El primer modelo no tiene ninguna variable independiente en su identificación -un modelo de sólo interceptos-. La varianza de "barrios" es de 0.82 . Esta varianza disminuye significativamente (0.493) al incorporar al modelo las variables de contexto (modelo 3). Esta reducción indica que las características de barrio están relacionadas estadísticamente con la varianza del nivel agregado. Sin embargo, la varianza

\section{Gráfica 1}

Varianzas estimadas e intervalos de confianza para el nivel de barrio en cuatro modelos multinivel

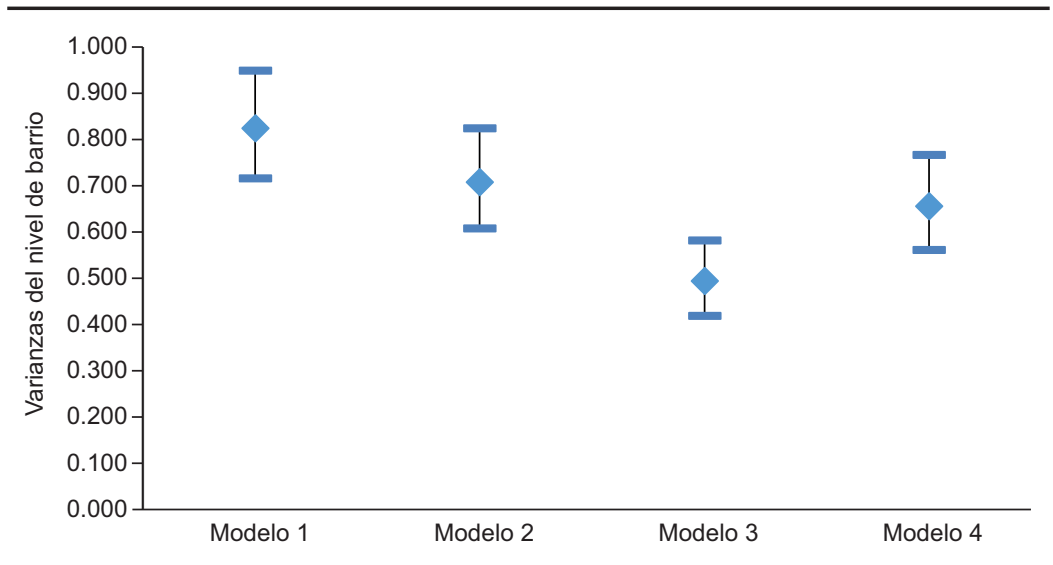

Nota: modelo 1: con sólo intercepto; modelo 2: con variables individuales; modelo 3: con variables de barrio; modelo 4: con todas las variables.

Fuente: Elaboración propia. 


\section{Cuadro 1}

Costa Rica. Personas de 12 a 17 años: estadísticas descriptivas de variables por incluir del modelo, según si son variables principales $\mathrm{o}$ adicionales, y si son de barrio o de nivel individual

\begin{tabular}{lcc}
\hline Variable & Promedio & Desv. est. \\
\hline Variable dependiente
\end{tabular}

Variable dependiente

Porcentaje de asistencia

Variables individuales $(n=27987)$

$\%$ mujeres 50.5

Índice económico promedio de jóvenes (escala 0 a 8)

$\%$ con discapacidad

Años promedio de escolaridad del jefe del hogar

Número promedio de menores de edad en el hogar

$\%$ en hogar con emigrante

\% jóvenes económicamente activos

$\%$ nacido en Nicaragua

$\%$ indígena

$\%$ migrantes internos en últimos 5 años

Variables de barrio $(n=1310)$

Índice económico promedio de barrio (escala 0 a 8) $\quad 4.2$

Escolaridad promedio de jefes $\quad 7.6$

Porcentaje promedio de hogares con emigrantes $\quad 2.7$

Porcentaje promedio de jóvenes económicamente activos $\quad 4.5$

Porcentaje promedio de jóvenes nicaragüenses $\quad 6.8$

Porcentaje promedio de jóvenes indígenas $\quad 1.9$

Porcentaje promedio de jóvenes migrantes internos $\quad 9.0$

$\%$ de barrios en zona rural

26.4

Fuente: INEC, Censo de Población, 2011.

vuelve a crecer en el modelo especificado con todas las variables, y no es significativamente distinta de la varianza del modelo 1. Esta nueva estimación sugiere que las variables individuales pesan más que las contextuales en la estimación de la varianza de los interceptos.

La anterior conclusión se refuerza con las estimaciones de los coeficientes de los modelos. De acuerdo con el Cuadro 2, casi todas las variables 


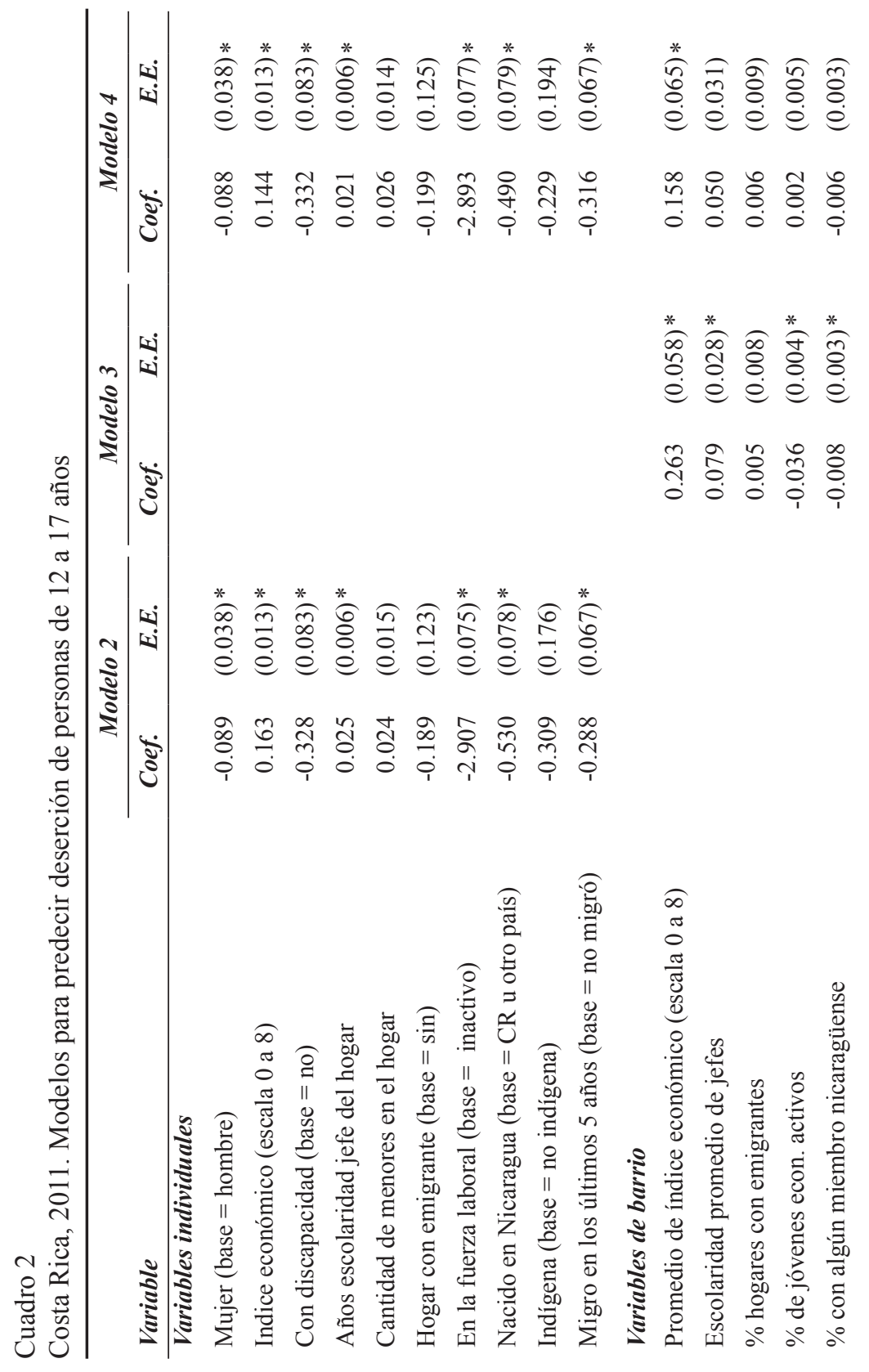




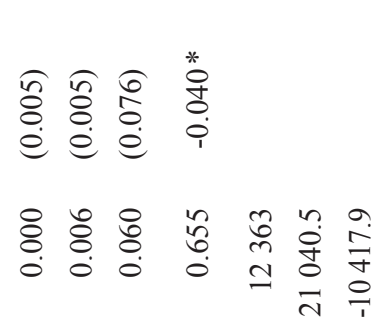

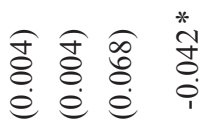

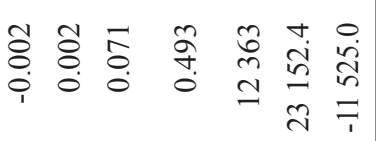

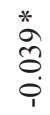

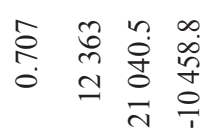

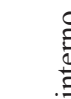

苞 苟

感

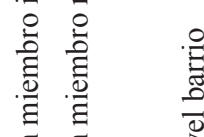

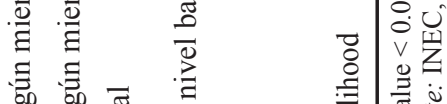

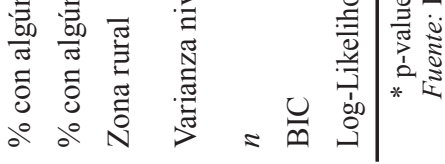


individuales predicen la asistencia a la secundaria, aun si se controla por las variables contextuales, no sólo porque sus coeficientes son significativamente distintos de cero, sino también por la magnitud de sus coeficientes. El modelo 3 se estima únicamente con las variables del barrio; según sus coeficientes, el nivel socioeconómico promedio del barrio y la escolaridad media de los jefes aumenta la probabilidad de que un joven que habita el barrio se mantenga asistiendo a la educación, mientras que los porcentajes de jóvenes ocupados o la proporción de nicaragüenses disminuye esta probabilidad. No obstante, cuando se incorporan las variables individuales, la mayoría de los coeficientes de éstas disminuyen su magnitud (en valor absoluto) y dejan de ser significativamente distintos de cero. La única covariable de barrio con un coeficiente considerablemente alto en el modelo 4 es la del índice socioeconómico del barrio.

El modelo 4 de interceptos aleatorios con variables individuales y contextuales es el escogido como modelo final. El Cuadro 3 contiene los coeficientes, sus errores estándar y los odds ratios o razones de momios. Además, para facilitar la interpretación de los resultados -más allá de los odds ratios-y seguir con las recomendaciones de la American Statistical Association (Wasserstein y Lazar, 2016) de interpretar la magnitud de los coeficientes de una regresión -y no utilizar las probabilidades asociadas (o $p$-values) como indicadores de la magnitud de un efecto, se estimaron probabilidades predichas con las variables más importantes según los objetivos del análisis (Gráficas 2 y 3 ). De acuerdo con los objetivos planteados, el resultado más importante es que el nivel socioeconómico individual del hogar del joven y el nivel socioeconómico del barrio predicen en forma separada la probabilidad de asistir a la secundaria. Por cada punto adicional en el índice económico del hogar del joven, los momios de la asistencia a la educación aumentan 15\%; además, por cada punto adicional en el índice del barrio, los momios aumentan $17 \%$. En términos de probabilidades predichas (Gráfica 2), se puede concluir que un joven con un índice económico de 1 tiene una probabilidad de estar asistiendo a la educación de 0.84 , mientras que, para un joven con un índice económico de 7 , esta probabilidad aumenta a 0.93 . Una diferencia similar se encuentra entre un joven que vive en un barrio con un índice económico de 1 y aquel que vive en un barrio con índice económico de 7 ( 0.83 vs. 0.93 ). Como comparación, nótese que el coeficiente para la variable escolaridad del jefe también es significativamente distinto de cero; sin embargo, en hogares con jefes con primaria completa (seis años de escolaridad), la probabilidad de asistencia del joven es de 0.89; en hogares con jefes con secundaria completa (la académica se completa con 11 años y la técnica con 12, por lo que se escogió como valor de referencia el 12), esta probabilidad apenas aumenta a 0.90 . 


\section{Cuadro 3}

Costa Rica, 2011. Modelo 4: modelo final de interceptos aleatorios y pendiente aleatoria para la variable del porcentaje de viviendas con TV por cable (continuación)

\begin{tabular}{|c|c|c|c|}
\hline & Coef. & E.E. & OR \\
\hline \multicolumn{4}{|l|}{ Variables individuales } \\
\hline Mujer (base $=$ hombre) & -0.088 & $-0.038 *$ & 0.92 \\
\hline Índice económico (escala 0 a 8) & 0.144 & $-0.013 *$ & 1.15 \\
\hline Con discapacidad $($ base $=$ no $)$ & -0.332 & $-0.083 *$ & 0.72 \\
\hline Años escolaridad jefe del hogar & 0.021 & $-0.006^{*}$ & 1.02 \\
\hline Cantidad de menores en el hogar & 0.026 & -0.014 & 1.03 \\
\hline Hogar con emigrante $($ base $=\sin )$ & -0.199 & -0.125 & 0.82 \\
\hline En la fuerza laboral (base $=$ inactivo) & -2.893 & $-0.077 *$ & 0.06 \\
\hline Nacido en Nicaragua (base $=\mathrm{CR} u$ otro país) & -0.490 & $-0.079 *$ & 0.61 \\
\hline Indígena (base = no indígena) & -0.229 & -0.194 & 0.80 \\
\hline Migro en los últimos 5 años (base $=$ no migró $)$ & -0.316 & $-0.067 *$ & 0.73 \\
\hline \multicolumn{4}{|l|}{ Variables de barrio } \\
\hline Promedio de índice económico (escala 0 a 8) & 0.158 & $-0.065 *$ & 1.17 \\
\hline Escolaridad promedio de jefes & 0.050 & -0.031 & 1.05 \\
\hline$\%$ hogares con emigrantes & 0.006 & -0.009 & 1.01 \\
\hline$\%$ de jóvenes econ. activos & 0.002 & -0.005 & 1.00 \\
\hline$\%$ con algún miembro nicaragüense & -0.006 & -0.003 & 0.99 \\
\hline$\%$ con algún miembro indígena & 0.000 & -0.005 & 1.00 \\
\hline$\%$ con algún miembro migrante interno & 0.006 & -0.005 & 1.01 \\
\hline Zona rural & 0.060 & -0.076 & 1.06 \\
\hline Constante & 0.450 & $-0.174 *$ & \\
\hline Varianza de interceptos & 0.655 & & \\
\hline Desv. estándar de interceptos & 0.810 & & \\
\hline Tamaño de muestra & 12363 & & \\
\hline $\mathrm{BIC}$ & 21040.52 & & \\
\hline Log. verosimilitud & -10417.87 & & \\
\hline
\end{tabular}

$*$ p-value $<0.05$.

Fuente: INEC, Censo de Población, 2011. 


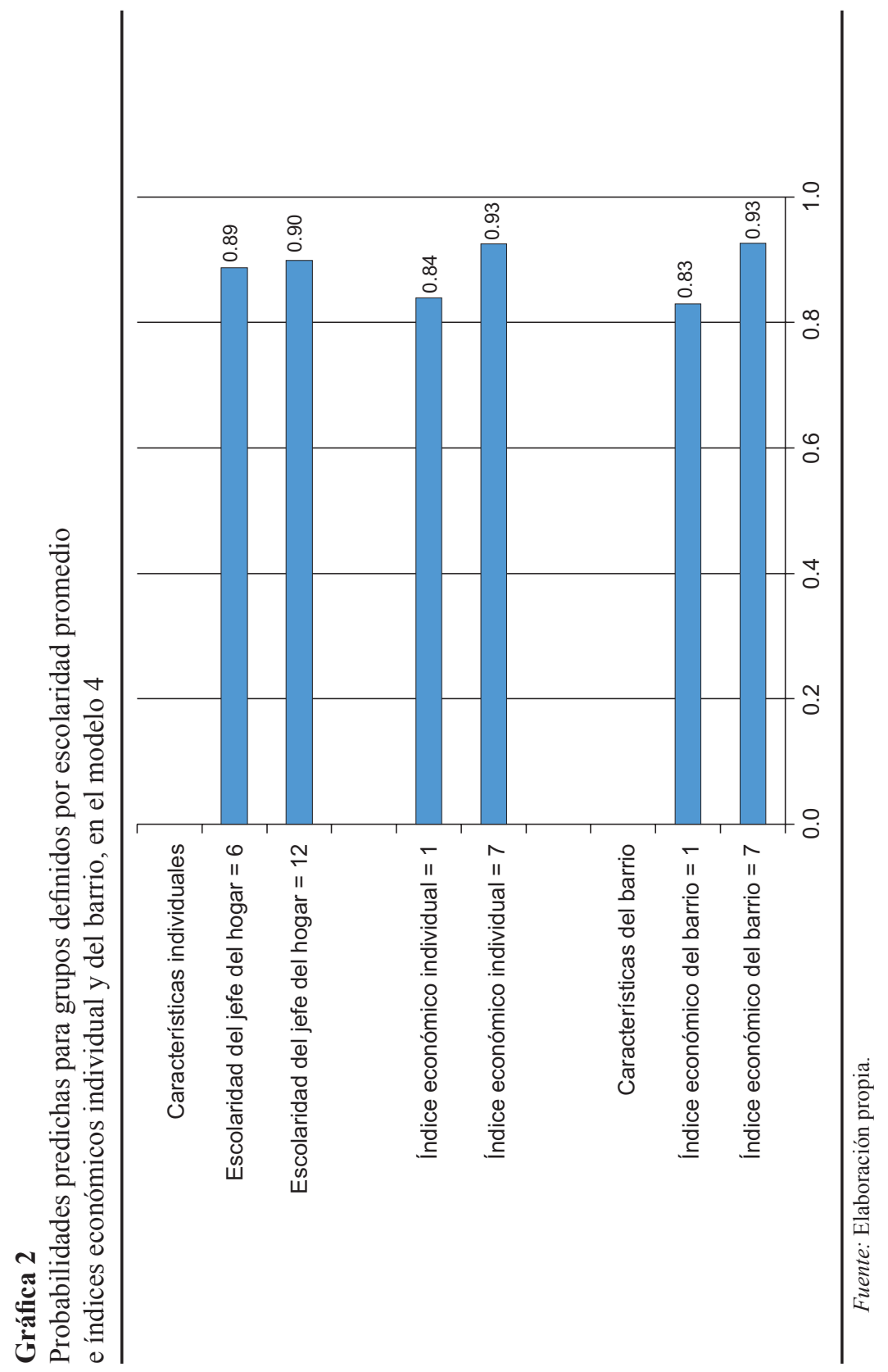




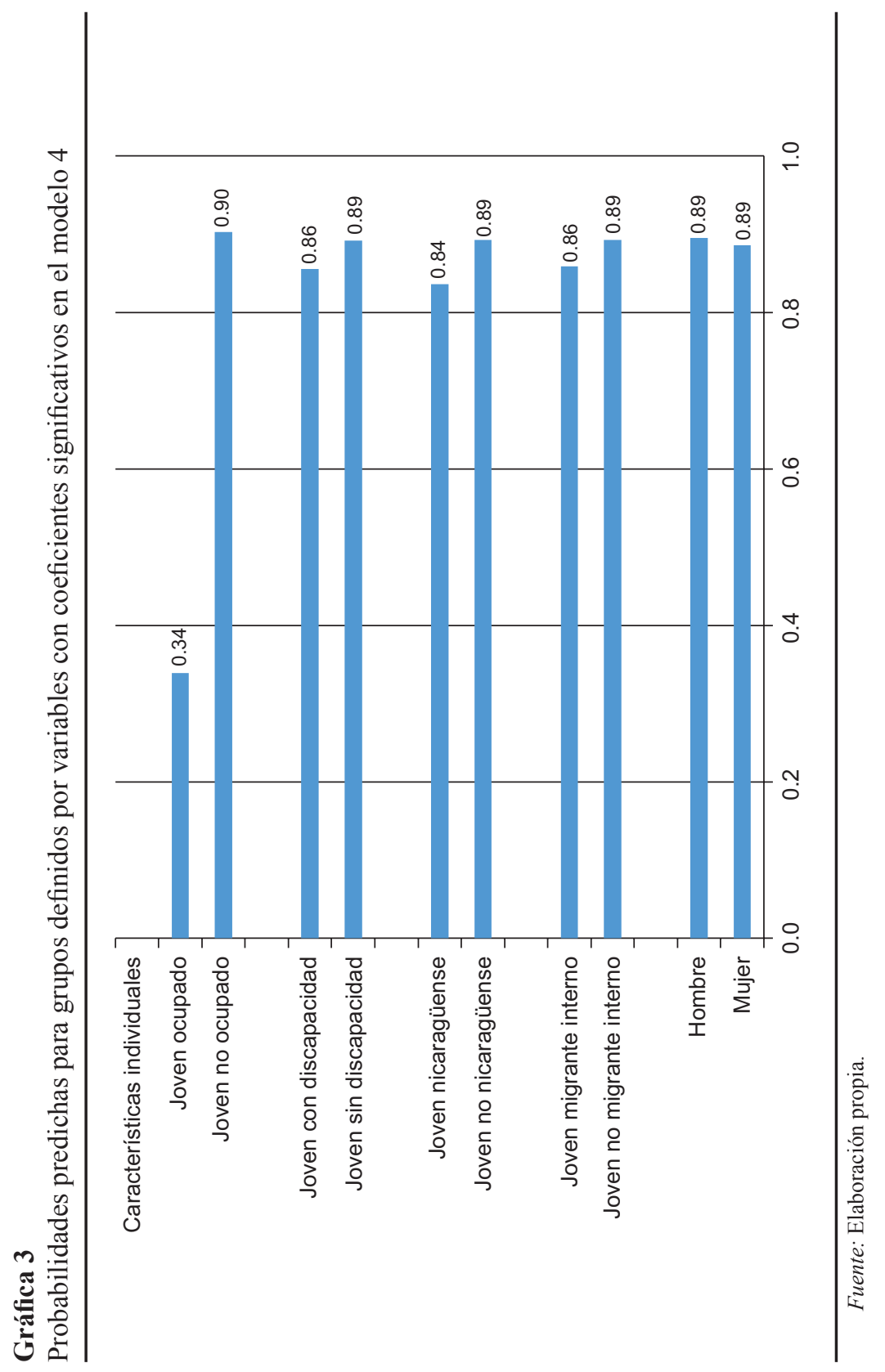


Las personas con discapacidad, los nicaragüenses y los que migraron recientemente a su actual municipio de residencia tienen menores probabilidades de asistir a la secundaria que aquellos jóvenes que no comparten estas características. De acuerdo con las probabilidades predichas, si se tiene alguna de estas características, la reducción en la probabilidad de permanecer en el sistema educativo es del orden de entre 3 y 5 puntos porcentuales (Gráfica 3). La variable que predice las mayores diferencias en asistencia es el estar en la fuerza laboral; si un joven está trabajando o buscando empleo, los momios de asistir a la secundaria se reducen en 94\%. En términos de probabilidades predichas, un joven en la fuerza laboral tiene una probabilidad de asistir a la educación de 0.34 , mientras que un joven inactivo económicamente tiene una probabilidad de 0.90 . Si bien es cierto que este resultado coincide con los hallazgos reportados por otros investigadores tanto en Costa Rica (Castro-Valverde, 2006; Herrera-Vargas, 2012; MoreiraMora, 2007) como en Latinoamérica en general (Alcázar, 2009; Aristimuño, 2009; Espíndola y León, 2002; Fernández, 2009; Román, 2009), recuérdese que el no contar con datos longitudinales, sino transversales, impide conocer si la inserción en el mercado laboral es previa o posterior a la decisión de no permanecer en el sistema escolar.

De acuerdo con el modelo 3, los momios de asistencia a la secundaria entre las mujeres son $8 \%$ menores a los momios de los hombres. Este resultado contradice las estadísticas oficiales (PEN, 2013) que señalan que el abandono es mayor entre los hombres que entre las mujeres. El resultado se puede deber a los criterios estrictos de inclusión que se plantearon en el análisis para asegurar que las personas analizadas fueran en realidad estudiantes de secundaria. Sin embargo, al observar las probabilidades predichas (Gráfica 3), se observa que la diferencia en asistencia entre hombres y mujeres no es sustantivamente diferente: 0.89 para ambos grupos.

Otro de los resultados aparentemente inesperados es que el modelo 4 señala que no hay diferencias significativas en los odds de asistir a la educación entre los jóvenes de zona urbana y los de zona rural. Tomando en cuenta el diseño de la muestra, se estimó un modelo (que no se presenta) con la variable dicotómica rural como única covariable. Se encuentra que los momios de estar en el sistema educativo entre los jóvenes rurales son $32 \%$ menores que los jóvenes de zonas urbanas. El hecho de que el odds ratio de dicha variable en el modelo 4 no sólo no sea significativamente distinto de uno sugiere que las diferencias por zona se explican por las otras variables individuales y contextuales incorporadas en el modelo 4. En otras palabras, comparados con los habitantes de zonas urbanas, los jóvenes de zonas rurales tienen menor nivel socioeconómico, habitan en hogares con jefes con 
menos años de escolaridad, trabajan en mayor proporción, y tienen mayor probabilidad de ser extranjeros (nicaragüenses), indígenas o migrantes internos.

\section{Conclusiones}

La mayoría de los estudios sobre asistencia al sistema escolar y rendimiento académico se centran en las características individuales de los estudiantes y las características de las instituciones educativas en forma singular y como parte de un sistema (Berns, 2012; Espíndola y León, 2002; Tyler y Lofstrom, 2009; Rumberger y Lim, 2008). Cuando se analiza el contexto de la problemática, los estudios enfatizan el rol que juega la familia en las motivaciones del estudiante para seguir estudiando (Herrera-Vargas, 2012; Moreira-Mora, 2007; Trejos, 2010). Los resultados de la presente investigación muestran que existen también factores de la comunidad que pueden estar asociados al progreso estudiantil. La mayoría de las variables del nivel comunal que están relacionadas con la asistencia a la secundaria son indicadores del nivel socioeconómico. Cuanto menor es el nivel socioeconómico del "barrio" -medido por la frecuencia de viviendas hechas con material de desecho (tugurios)-, menor es la probabilidad de que un joven permanezca en el sistema educativo, controlando por el nivel socioeconómico del hogar. Estos hallazgos coinciden con otras investigaciones en otros países (Kling, Liebman y Katz, 2007; Patterson, 2008; Rendón, 2014; Wodtke, Harding y Elwert, 2011), y también coinciden con los reportados en la presente investigación. Con un índice económico construido a partir de las características de las viviendas, se encontró que en Costa Rica tanto el nivel socioeconómico individual como el del barrio tienen una asociación positiva con la probabilidad de que el joven esté en la educación secundaria. El hallazgo es similar al reportado por Solís y Puga (2011) para México, aunque -al construir su propio índiceestos autores no sólo utilizan características físicas de la vivienda, sino también variables de ingreso y escolaridad. La principal limitación del análisis para Costa Rica es que el censo no cuenta con variables de ingreso, por lo que el nivel socioeconómico se aproxima según las características físicas de la vivienda.

Llama la atención además que el índice económico del barrio (que aproxima mejor el nivel de riqueza por estar construido a partir de características físicas) esté asociado con la asistencia, pero la escolaridad promedio del barrio no lo esté. Según la perspectiva de Coleman (1988), el nivel educativo de las redes en las que se circunscriben los estudiantes denota la 
calidad del capital social disponible para los estudiantes y sus familias. Los resultados del presente análisis sugieren que es la afluencia o los recursos monetarios de los habitantes del barrio, más que sus características educativas, los que componen el capital social del joven y favorecen su permanencia en la secundaria. De esta forma, los resultados responderían mejor a la perspectiva de Bourdieu (1986), en el sentido de que el capital social se utiliza por las clases dominantes para mantener la reproducción del sistema. En este sentido, las comunidades con pobres características físicas -viviendas construidas con material de desecho, propiedades invadidas o en precario, y falta de activos (restricciones físicas a las actividades rutinarias, en la explicación de Solís y Puga, 2011)- plantean mayores dificultades a los jóvenes para que accedan a un capital social comunitario que les permita o estimule a seguir estudiando, reproduciendo así las desigualdades existentes.

Pese a que el análisis mostró la importancia del nivel económico del barrio como variable predictora, la mayoría de las variables que predicen la asistencia a la secundaria son las individuales. La participación económica del joven disminuye fuertemente la probabilidad que tiene de estar estudiando, independientemente del nivel económico del hogar y del barrio. Sin embargo, al combinar estas tres variables, se encontraría que los jóvenes que están laborando y que al mismo tiempo provienen de contextos familiares y residenciales de bajos ingresos estarían enfrentándose en mayor proporción a la exclusión por parte del sistema educativo. Si además estos jóvenes tienen discapacidad o son nicaragüenses o migrantes internos (una combinación altamente plausible), los problemas de exclusión se acrecientan (Eamon, 2005; Espíndola y León, 2002; Mora Salas y Pérez Sáinz, 2009; Pong y Hao, 2007; Portes y McLeod, 1996; Terigi, 2009).

En general, el estudio mostró que sí hay posibles "efectos de barrio" sobre la deserción estudiantil en secundaria en Costa Rica. La magnitud de estas asociaciones es baja; pesan más los factores individuales que los contextuales. Sin embargo, no se pueden descartar, en particular la afluencia o nivel económico "físico" de la comunidad en la que se habita. La importancia de la comunidad es conocida por los tomadores de decisiones en Costa Rica. A mediados de la década pasada, el Consejo Superior de Educación promovió un Acuerdo Nacional sobre Educación en el que se mencionaba la "participación protagónica de la comunidad y la familia en el proceso educativo" (PEN, 2008, p. 79). Las políticas educativas tienen que enfocarse también en motivar el involucramiento de los vecinos en los esfuerzos de las instituciones educativas para retener a los estudiantes y aumentar el rendimiento escolar de los jóvenes. Es importante reconocer que las políticas educativas no pueden alterar fácilmente las características socioeconómicas 
de un barrio, pero sí pueden promover el trabajo conjunto entre las instituciones educativas y su comunidad circundante.

\section{Bibliografía}

Agnew, J. (1989). The devaluation of place in social science. En J. Agnew y J. Duncan (eds.), The power of place: Bringing together geographical and sociological imaginations. Londres: Routledge.

Alcázar, L. (2009). Asistencia y deserción en escuelas secundarias rurales del Perú. REICE. Revista Iberoamericana sobre Calidad, Eficacia y Cambio en Educación, 7(4), 136-163. Recuperado de http://www.reda lyc.org/pdf/551/55114094008.pdf

Alonso Meneses, G. y Ángeles Salinas, C. A. (2014). La juventud mixteca en Tijuana: educación, desarrollo, discriminación y neo-indianidad. Frontera Norte. Revista Internacional de Fronteras, Territorios y Regiones, 26(51), 25-52. Recuperado de https://fronteranorte.colef.mx/ index.php/frontera norte/article/view/555/97

Arguedas-Negrini, I. y Jiménez-Segura, F. (2007). Factores que promueven la permanencia de estudiantes en la educación secundaria. Revista Electrónica Actualidades Investigativas en Educación, 7(3), 1-36. Recuperado de http://www.redalyc.org/articulo.oa?id=44770317

Aristimuño, A. (2009). El abandono de los estudios del nivel medio en Uruguay: un problema complejo y persistente. REICE. Revista Iberoamericana sobre Calidad, Eficacia y Cambio en Educación, 7(4), 180-197. Recuperado de https://revistas.uam.es/index.php/reice/article/view/ 5388/5827

Baronnet, B. (2013). Lenguas y participación comunitaria en la educación indígena en México. AIBR. Revista de Antropología Iberoamericana, 8(2), 183-208. Recuperado de http://www.redalyc.org/pdf/623/62329 867003.pdf

Berns, R. (2012). Child, family, school, community: Socialization and support. Boston, MA: Cengage Learning (Psychology of the Exceptional Child Series).

Bourdieu, P. (1986). The forms of capital. En J. Richardson (ed.), Handbook of theory and research for the sociology of education (pp. 241-258). Westport, CT: Greenwood Press.

Brenes-Camacho, G. (2014). Factores contextuales de la comunidad asociados con repitencia, deserción y reprobación en las escuelas y colegios de Costa Rica, Quinto Informe del Estado de la Educación. San José, Costa Rica: Programa Estado de la Nación, Consejo Nacional de Rec- 
tores. Recuperado de: http://www.estadonacion.or.cr/files/biblioteca virtual/educacion/005/Gilbert_Brenes_Factores_contextuales_de_ la\%20_comunidad.pdf

Bronfenbrenner, U. (2005). Making human beings human: Bioecological perspectives on human development. Ithaca, NY: Cornell University / SAGE.

Callaghan, E. y Colton, J. (2008). Building sustainable and resilient communities: A balancing of community capital. Environment, Development and Sustainability, 10(6), 931-942. Recuperado de https://link.springer. com/article/10.1007/s10668-007-9093-4

Cardozo, S. (2010). El comienzo del fin: las decisiones de abandono durante la educación media y su incidencia en las trayectorias. En T. Fernández Aguerre (coord.), La desafiliación en la educación media y superior de Uruguay: conceptos, estudios y políticas (pp. 65-82). Montevideo: Comisión Sectorial de Investigación Científica. Universidad de la República.

Castro Valverde, C. (2006). Educación. Duodécimo Informe Estado de la Nación en Desarrollo Humano Sostenible. San José, Costa Rica: Programa Estado de la Nación, Consejo Nacional de Rectores. Recuperado de: http://estadonacion.or.cr/files/biblioteca_virtual/012/Castro_2006. pdf

Coleman, J. (1988). Social capital in the creation of human capital. The American Journal of Sociology, 94 (suplemento), S95-S120. Recuperado de https://faculty.washington.edu/matsueda/courses/587/readings/ Coleman\%201988.pdf

Cueto, S. (2004). Factores predictivos del rendimiento escolar, deserción e ingreso a educación secundaria en una muestra de estudiantes de zonas rurales del Perú. Archivos Analíticos de Políticas Educativas, 12(35), 1-42 Recuperado de http://repositorio.minedu.gob.pe/handle/123456789/ 150

Dika, S. y Singh, K. (2002). Applications of social capital in educational literature: A critical synthesis. Review of Educational Research, 72(1), 31-60. Recuperado de http://journals.sagepub.com/doi/pdf/10.3102/0034 6543072001031

Domingos, A. M. (1989). Influence of the social context of the school on the teacher's pedagogic practice. British Journal of Sociology of Education, 10(3), 351-366. Recuperado de https://www.jstor.org/stable/1392743? seq=1\#page scan tab_contents

Eamon, M. (2005). Social-demographic, school, neighborhood, and parenting influences on the academic achievement of latino young adolescents. 
Journal of Youth and Adolescence, 34(2), 163-174. Recuperado de https:// link.springer.com/content/pdf/10.1007\%2Fs10964-005-3214-x.pdf

Espíndola, E. y León, A. (2002). La deserción escolar en América Latina: un tema prioritario para la agenda regional. Revista Iberoamericana de Educación, 30, 36-62 Recuperado de https://rieoei.org/RIE/article/ view/941

Fernández, T. (2009). La desafiliación en la educación media en Uruguay. Una aproximación con base en el panel de estudiantes evaluados por PISA 2003. REICE. Revista Iberoamericana sobre Calidad, Eficacia y Cambio en Educación, 7(4), 164-179. Recuperado de http://www.redalyc. org/pdf $/ 551 / 55114094009$.pdf

Fernández, T. (2010a). Factores escolares y desafiliación en la enseñanza media superior de Uruguay (2003-2007). En T. Fernández Aguerre (coord.), La desafiliación en la educación media y superior de Uruguay: conceptos, estudios y políticas (pp. 99-121). Montevideo: Comisión Sectorial de Investigación Científica, Universidad de la República.

Fernández, T. (2010b). Incidencia y trayectorias de desafiliación. En T. Fernández Aguerre (coord.), La desafiliación en la educación media y superior de Uruguay: conceptos, estudios y políticas (pp. 51-63). Montevideo: Comisión Sectorial de Investigación Científica, Universidad de la República.

Hallinger, P. y Murphy, J. (1986). The social context of effective schools. American Journal of Education, 94(3), 328-355. Recuperado de https:// www.journals.uchicago.edu/doi/10.1086/443853

Herrera-Vargas, L. (2012). Principales causas de deserción estudiantil y técnicas aplicadas para su prevención desde la gestión en el Colegio Nocturno La Unión y en el Colegio Nacional Virtual Marco Tulio Salazar, sede en el cantón de La Unión. Revista Gestión de la Educación, 2(2), 1-34. Recuperado de https://revistas.ucr.ac.cr/index.php/gestedu/ article/view/5865/8139

INEC (Instituto Nacional de Estadística y Censos) (2011). Costa Rica: X Censo Nacional de Población y VI de Vivienda, Censo 2011. San José, Costa Rica: Instituto Nacional de Estadística y Censos.

Kling, J., Liebman, J. y Katz, L. (2007). Experimental analysis of neighborhood effects. Econometrica, 75(1), 83-119. Recuperado de https://online library.wiley.com/doi/epdf/10.1111/j.1468-0262.2007.00733.x

Kreft, I. y De Leeuw, J. (1998). Introducing multilevel modeling. Londres: Sage.

Li, J., Dow, W. y Rosero-Bixby, L. (2014). The declining effect of sibling size on children's education in Costa Rica. Demographic Research, 31, 
1431-1454. Recuperado de https://www.jstor.org/stable/26350102?seq= 1\#page_scan_tab_contents

Lizasoain, L., Joaristi, L., Lukas, J. F. y Santiago, K. (2007). Contextual effects of socioeconomic level on academic achievement in obligatory secondary education in the Basque Autonomous Community (Spain). Differential study about socioeconomic level of families and school centers. Education Policy Analysis Archives, 15(8), 1-35. Recuperado de https://epaa.asu.edu/ojs/article/view/56

Montero, E., Rojas, S., Zamora, E. y Rodino, A. M. (2012). Costa Rica en las pruebas PISA 2009 de Competencia Lectora y Alfabetización Matemática. Cuarto Informe del Estado de la Educación, Informe final. San José, Costa Rica: Programa Estado de la Nación, Estado de la Educación. Recuperado de: http:/estadonacion.or.cr/files/biblioteca_virtual/educacion/ 004/montero\%20et\%20al_pisa\%20competencia\%20lectora $\% 20 y \% 20$ alfabetizacion $\% 20$ matematica.pdf

Mora Salas, M. y Pérez Sáinz, J. P. (2009). Se acabó la pura vida. Amenazas y desafios sociales en la Costa Rica del siglo XXI. San José, Costa Rica: Flacso.

Moreira-Mora, T. (2007). Perfil sociodemográfico y académico de estudiantes en deserción del sistema educativo. Actualidades en Psicología, 21(108), 145-165. Recuperado de http://www.redalyc.org/articulo.oa?id= 133212641007

Murillo López, S. (2005). Etnicidad, asistencia escolar y trabajo de niños y jóvenes rurales en Oaxaca. En M. Mier y Terán y C. Rabell (coords.), Jóvenes y niños. Un enfoque sociodemográfico (pp. 249-288). Ciudad de México: Flacso / UNAM / Miguel Ángel Porrúa.

Patterson, R. (2008). Neighborhood effects on high-school drop-out rates and teenage childbearing (Research Papers, 08-12.). Center for Economic Studies, CES. Recuperado de https://papers.ssrn.com/sol3/papers. cfm?abstract_id $=1134940$

PEN (Programa Estado de la Nación) (2005). Primer Informe Estado de la Educación. San José, Costa Rica: Programa Estado de la Nación, Consejo Nacional de Rectores.

PEN (Programa Estado de la Nación) (2008). Segundo Informe Estado de la Educación. San José, Costa Rica: Programa Estado de la Nación, Consejo Nacional de Rectores.

PEN (Programa Estado de la Nación) (2011). Tercer Informe Estado de la Educación. San José, Costa Rica: Programa Estado de la Nación, Consejo Nacional de Rectores.

PEN (Programa Estado de la Nación) (2013). Cuarto Informe Estado de la 
Educación. San José, Costa Rica: Programa Estado de la Nación, Consejo Nacional de Rectores.

Pong, S. y Hao, L. (2007). Neighborhood and school factors in the school performance of immigrants' children. International Migration Review, 41(1), 206-241. Recuperado de https://onlinelibrary.wiley.com/doi/ epdf/10.1111/j.1747-7379.2007.00062.x

Portes, A. (2000). The two meanings of social capital. Sociological Forum, 15(1). 1-12. Recuperado de https://www.jstor.org/stable/3070334?seq=1 \#page_scan_tab_contents

Portes, A. y MacLeod, D. (1996). Educational progress of children of immigrants: The roles of class, ethnicity, and school context. Sociology of Education, 69(4), 255-275. Recuperado de https://www.jstor.org/stable/ 2112714 ?seq=1\#page_scan_tab_contents

Putnam, R. (1993). The prosperous community: Social capital and public life. The American Prospect, 13, 35-42. Recuperado de http://prospect. org/article/prosperous-community-social-capital-and-public-life

Rendón, M. (2014). Drop out and disconnected young adults: Examining the impact of neighborhood and school contexts. The Urban Review, 46(2), 169-196. Recuperado de https://link.springer.com/article/10.1007/ s11256-013-0251-8

Rodríguez, E. (2012). ¿Barreras lingüísticas en la educación? La influencia de la lengua materna en la deserción escolar. Revista Economía, 35(69), 83-151. Recuperado de http://revistas.pucp.edu.pe/index.php/economia/ article/view/2711

Rodríguez Romero, J. (2016). Factores de deserción del estudiantado migrante nicaragüense en el Liceo Rural San Julián. Estudio de caso: Liceo Rural San Julián en el contexto fronterizo de Sarapiquí, Costa Rica (2003-2010). Revista Perspectivas: Estudios Sociales y Educación Cívica, 13, 13-45. Recuperado de http://www.revistas.una.ac.cr/index.php/ perspectivas/article/view/8987/10322

Román, M. (2009). El fracaso escolar de los jóvenes en la enseñanza media. ¿Quiénes y por qué abandonan definitivamente el liceo en Chile? REICE. Revista Iberoamericana sobre Calidad, Eficacia y Cambio en Educación, 7(4), 95-119. Recuperado de http://www.redalyc.org/pdf/551/5511 4094006.pdf

Ruiz-Guevara, L., Castro-Pérez, M. y León-Sáenz, A. T. (2010). Transición a la secundaria: los temores y preocupaciones que experimentan los estudiantes de primaria. Revista Iberoamericana de Educación, 52(3). 1-13 Recuperado de https://rieoei.org/RIE/article/view/1793

Rumberger, R. y Lim, S. A. (2008). Why students drop out of school: A review 
of 25 years of research (California Dropout Research Project Report, 15). Santa Barbara, CA: University of California. Recuperado de https:// www.issuelab.org/resources/11658/11658.pdf

Solís, P. y Puga, I. (2011). Efectos del nivel socioeconómico de la zona de residencia sobre el proceso de estratificación social en Monterrey. Estudios Demográficos y Urbanos, 26(2), 233-265. Recuperado de https:// estudiosdemograficosyurbanos.colmex.mx/index.php/edu/article/ view/1383/13

Sunkel, G. (2006). El papel de la familia en la protección social en América Latina. Santiago de Chile: Comisión Económica para América Latina (CEPAL) (Políticas Sociales, 120).

Terigi, F. (2009). Segmentación urbana y educación en América Latina. Aportes de seis estudios sobre políticas de inclusión educativa en seis grandes ciudades de la región. REICE. Revista Iberoamericana sobre Calidad, Eficacia y Cambio en Educación, 7(4), 28-47. Recuperado de http://www.redalyc.org/articulo.oa?id=55114094003

Tijoux, M. E. (2013). Las escuelas de la inmigración en la ciudad de Santiago: elementos para una educación contra el racismo. Polis. Revista Latinoamericana, 35, 1-17. Recuperado de https://journals.openedition. org/polis/9338

Trejos, J. D. (2010). Indicadores sobre equidad en la educación para Costa Rica. Tercer Informe Estado de la Educación. Informe final. San José, Costa Rica: Programa Estado de la Nación. Recuperado de: http://esta donacion.or.cr/files/biblioteca_virtual/educacion/003/Trejos_2010_ Indicadores_Equidad_Educacion.pdf (17 de mayo de 2016).

Tyler, J. y Lofstrom, M. (2009). Finishing high school: Alternative pathways and dropout recovery. The Future of Children, 19(1), 77-103. Recuperado de https://files.eric.ed.gov/fulltext/EJ842053.pdf

Vargas, J. y Slon, P (2012). ¿Protegen los programas sociales de la deserción educativa en Costa Rica? Cuarto Informe del Estado de la Educación. San José, Costa Rica: Programa Estado de la Nación. Consejo Nacional de Rectores. Recuperado de http://www.estadonacion.or.cr/files/biblioteca _virtual/educacion/004/slon-y-vargas-desercion-educativa.pdf

Wasserstein, R. y Lazar, N. (2016). The ASA's statement on p-values: Context, process, and purpose. The American Statistician, 70(2): 129-133. Recuperado de http://amstat.tandfonline.com/doi/full/10.1080/000313 05.2016.1154108

Watson, J., Wright, S., Allen, J., Hay, I., Cranston, N. y Beswick, K. (2017). Increasing students' social capital through community involvement in rural and regional education. Australian and International Journal of 
Rural Education, 27(3), 142-157. Recuperado de https://research-repo sitory.griffith.edu.au/bitstream/handle/10072/354141/WatsonPUB4182. pdf? sequence $=1$

Wodtke, G., Harding, D. y Elwert, F. (2011). Neighborhood effects in temporal perspective: The impact of long-term exposure to concentrated disadvantage on high school graduation. American Sociological Review, 76(5), 713-736. Recuperado de http://journals.sagepub.com/doi/ pdf $/ 10.1177 / 0003122411420816$

Wong, G. y Mason, W. (1985). The hierarchical logistic regression model for multilevel analysis. Journal of the American Statistical Association, 80(391), 513-524. Recuperado de https://www.jstor.org/stable/2288464? seq=1\#page_scan_tab_contents

\section{Acerca del autor}

Gilbert Brenes Camacho es doctor en Sociología y maestro en Salud de la Población por la Universidad de Wisconsin-Madison. Es profesor catedrático de la Escuela de Estadística y director del Centro Centroamericano de Población (periodo 2017-2021) de la Universidad de Costa Rica. Fue investigador y, durante dos años, coordinador nacional del proyecto CRELES: "Costa Rica: Estudio de Longevidad y Envejecimiento Saludable". Imparte cursos sobre análisis de sobrevida y modelos lineales generalizados. Su investigación se centra en la relación entre las instituciones públicas y los comportamientos demográficos de costarricenses y latinoamericanos, especialmente en el campo de la salud, el envejecimiento y la migración. ORCID: https://orcid.org/0000-0002-3163-7018

Entre sus publicaciones destacan:

Brenes Camacho, G. (2018). Number of children in a household and child well-being. Revista Latinoamericana de Población, 12(22), 5-31.

Brenes-Camacho, G. (2018). Retirement decisions and family characteristics in Costa Rica. Revista Latinoamericana de Familia, 10(1), 31-50.

Brenes-Camacho, G. (2011). Favourable changes in economic well-being and self-rated health among the elderly. Social Science \& Medicine, 72(8), 1228-1235.

Recepción: 28 de junio de 2016.

Aceptación: 7 de abril de 2018. 
